\title{
Un compromiso para la transformación social: el plan UC para consolidar a A+S en América Latina
}

\author{
F. Del Valle \\ R. Fontana \\ J. Sepúlveda \\ Pontificia Universidad Católica de Chile
}

\section{Resumen}

En marzo de 2021, la Pontificia Universidad Católica de Chile (UC) inició su plan de acompañamiento regional en el marco del proyecto Uniservitate en América Latina. Este proyecto global pretende institucionalizar y potenciar la implementación de la metodología de aprendizaje servicio $(A+S)$ en universidades católicas del continente, en coherencia con su misión institucional promoviendo así una educación integral que permita formar ciudadanos socialmente responsables. El objetivo del artículo es mostrar las etapas del plan de acompañamiento de la UC para las universidades Católica de Argentina, Católica de Pernambuco, Pontificia Javeriana de Bogotá y Católica de Ecuador. El plan de acompañamiento para institucionalizar el aprendizajeservicio en el continente posee un diseño definido con elementos fuertes en la formación, el acompañamiento y la evaluación siendo estos los ejes para una primera etapa de implementación en las universidades acompañadas. El aprendizaje-servicio es una metodología que busca desarrollar una educación integral a través de la formación de la responsabilidad social en el estudiantado y su institucionalización representa una oportunidad para que las universidades profundicen su contribución a una sociedad más justa y equitativa.

\section{Palabras clave}

Institucionalización, metodología activa, formación integral, educación superior.

Fecha de recepción: 24/XI/2021

Fecha de aceptación: 9/XII/2021 


\title{
A commitment to social transformation: the UC plan to consolidate service-learning in Latin America
}

\begin{abstract}
In March 2021, the Pontificia Universidad Católica de Chile (UC) began its regional support plan within the framework of the Uniservitate project in Latin America. This global project aims to institutionalize and promote the implementation of the service learning methodology in Catholic universities on the continent, in coherence with its institutional mission, thus promoting a comprehensive education that allows the formation of socially responsible citizens. The objective of the article is to show the stages of the UC accompaniment plan for the Catholic universities of Argentina, Catholic of Pernambuco, Pontificia Javeriana of Bogotá and Catholic of Ecuador. The accompaniment plan to institutionalize service-learning in the continent has a defined design with strong elements in training, accompaniment and evaluation, these being the axes for a first stage of implementation in the supervised universities. Servicelearning is a methodology that seeks to develop an integral education through the formation of social responsibility in the student body and its institutionalization represents an opportunity for universities to deepen their contribution to a more just and equitable society.
\end{abstract}

\section{Key words}

Institutionalization, active methodology, comprehensive training, higher education. 


\section{Un compromís per a la transformació social: el pla UC per consolidar I'aprenentatge servei a Amèrica Llatina}

\section{Resum}

El març de 2021, la Pontifícia Universidad Católica de Xile (UC) va iniciar el seu pla d'acompanyament regional en el marc del projecte Uniservitate a Amèrica Llatina. Aquest projecte global pretén institucionalitzar i potenciar la implementació de la metodologia d'aprenentatge servei $(A+S)$ en universitats catòliques del continent, en coherència amb la seva missió institucional promovent així una educació integral que permeti formar ciutadans socialment responsables. L'objectiu de l'article és mostrar les etapes del pla d'acompanyament de la UC per a les universitats Católica de Argentina, Católica de Pernambuco, Pontifícia Javeriana de Bogotá i Católica de l'Equador. El pla d'acompanyament per institucionalitzar l'aprenentatge servei al continent té un disseny definit amb elements forts en la formació, l'acompanyament i l'avaluació, i aquests són els eixos per a una primera etapa d'implementació a les universitats acompanyades. L'aprenentatge servei és una metodologia que busca desenvolupar una educació integral a través de la formació de la responsabilitat social en els estudiants i la seva institucionalització representa una oportunitat perquè les universitats aprofundeixin la seva contribució a una societat més justa i equitativa.

\section{Paraules clau}

Institucionalització, metodologia activa, formació integral, educació superior. 


\section{Introducción}

La Pontificia Universidad Católica de Chile (UC) participa del proyecto Uniservitate en su rol como nodo representante para América Latina, en donde una de las responsabilidades asociadas es la de elaborar un plan de acompañamiento regional junto a cuatro universidades católicas de la región, que durante el año 2020 se someten a un proceso de selección, quedando posteriormente entre las calificadas para el proyecto, en donde se busca fortalecer el proceso de institucionalización e implementación de aprendizaje-servicio. Esta metodología activa se define, según Furco y Billig (2002), como una metodología experiencial que integra actividades de servicio a la comunidad con el currículum académico, permitiendo que el estudiantado adquiera contenidos y herramientas que permitan trabajar necesidades genuinas de la comunidad. En ese contexto, surge la inquietud, ¿cómo se desarrolla un plan de institucionalización de aprendizaje-servicio en una Institución de Educación Superior Católica (IESC)? Como respuesta a la interrogante planteada, es que como nodo regional surge la propuesta de fortalecer este proceso en base a un modelo de institucionalización de aprendizajeservicio, entendido como un proceso que culmina con la etapa donde el aprendizaje-servicio queda integrado a la estructura de la universidad (Jouannet et al., 2015). Este artículo busca socializar las principales etapas del plan de acompañamiento de la UC y su co-diseño junto a las universidades asociadas al proyecto las cuales son: Universidad Católica de Argentina (UCA), Universidad Católica de Pernambuco (UNICAP), Pontificia Universidad Javeriana de Bogotá (PUJ) y Universidad Católica de Ecuador (PUCE). En este proceso en particular, las instituciones participantes autoaplicaron la rúbrica diseñada por el académico Andrew Furco (2011), con la diferencia de que ésta fue adaptada a la institucionalización del aprendizajeservicio en la UC. En base a este diagnóstico se desarrolló el plan de acompañamiento, que se divide en cuatro etapas: Lineamientos, Formación, Implementación y Seguimiento, y Evaluación.

En este artículo se buscará describir el proceso de desarrollo del plan de institucionalización de aprendizajeservicio en las Instituciones de Educación Superior Católica (IESC) que son parte del programa de acompañamiento de Uniservitate, el estado actual en la implementación de este plan, los procesos involucrados, además de las proyecciones que esperamos de esta implementación. A su vez, se revisará la metodología utilizada para realizar el diagnóstico, su aplicación y el desarrollo del plan, junto a sus principales hitos. Cabe mencionar que este artículo utiliza un enfoque metodológico de tipo cualitativo descriptivo.

\section{Metodología}

Para el cumplimiento de los objetivos de este artículo, se desarrolló una metodología cualitativa descriptiva que

Del Valle, F., Fontana, R. y Sepúlveda, J. (2021). Un compromiso para la transformación social: el plan UC para consolidar a A+S en América Latina. RIDAS, Revista Iberoamericana de Aprendizaje Servicio, 12, 80-90. DOI10.1344/RIDAS2021.12.9 
divide el proceso en 3 etapas.

Primero, a inicios del año 2021, previo a la propuesta del Plan de Acompañamiento se solicita realizar una autoevaluación en los equipos de cada universidad participante, utilizando la rúbrica de institucionalización de aprendizajeservicio UC en una carrera, instrumento que fue adaptado a partir de la rúbrica de Furco (2011) y que incluye aristas específicas para el desarrollo de este proyecto. Esta autoevaluación fue respondida por los equipos coordinadores de las 4 instituciones seleccionadas para este período. Además, la rúbrica incorporó una sección de preguntas abiertas sobre las principales temáticas a trabajar en el Plan de Acompañamiento. Según Caire et al., (2019) la rúbrica tiene como objetivo:

...orientar a cada carrera en el
diagnóstico del nivel de
institucionalización de
aprendizaje-servicio. Frente a lo
anterior, la rúbrica tiene las
siguientes dimensiones: 1)
Incorporación de aprendizaje-
servicio desde un enfoque
curricular; 2) Docentes
aprendizaje-servicio; 3) Trabajo
con socios comunitarios; 4)
Protagonismo Estudiantil; y 5)
Soporte y gestión de la
implementación de aprendizaje-
servicio en la unidad académica
(p. 59).

Al igual que el instrumento de Furco, la rúbrica de la UC incorpora 3 momentos de avance de la institucionalización de aprendizaje-servicio en cada subdimensión: Inicio (1), En proceso (2) y en consolidación (3) (Caire, et al., 2018). En el caso de la UC, la unidad académica se refiere a facultad, instituto o departamento que tiene a cargo la gestión de una o más carreras. Segundo, entre enero y febrero del 2021 , se realizó una sistematización de los principales mecanismos y acciones realizadas por el equipo del Programa aprendizaje-servicio UC, para acompañar y/o asesorar a instituciones de educación superior en la implementación de aprendizajeservicio. Esta sistematización permitió generar la estructura principal del Plan que fue presentada a los equipos coordinadores en las universidades acompañadas, propuesta que tiene el espíritu de ser un espacio de coconstrucción y aprendizaje mutuo. Parte de las decisiones que involucra el ejercicio diagnóstico efectuado con las universidades acompañadas, permite entregar lineamientos generales que propician la flexibilidad y adaptación del modelo a proponer, a las realidades, recursos y formas de ejecutar proyectos de cada universidad, respondiendo a sus necesidades, propósitos y cultura que poseen como institución de educación superior. No olvidar que parte importante de este ejercicio involucra el responder al perfil de egreso, misión y visión que cada universidad católica afronta en su contexto. Tercero, en marzo del año 2021, se realizó una instancia de diálogo que tenía como objetivo generar un compromiso regional que impulsara la implementación y el fortalecimiento de la metodología en el continente aportando a los desafíos que comparten los países que están siendo 
parte del proyecto: desigualdad, pobreza, equidad de género, fortalecimiento de las comunidades, entre otros. Lo anterior se relaciona con una de las acciones que gatilla lo analizado tras el diagnóstico aplicado y los resultados que arroja el mismo, nuestra convicción principal es la de concebir al aprendizaje-servicio como una de las vías desde la educación que transforma a las personas, la ciudadanía y por ende las sociedades bajo un prisma del traspaso de saberes y experiencias, una educación desde lo colectivo, con acción.

\section{Resultados y discusión}

Frente a lo anteriormente mencionado, los hitos que se componen como parte de los resultados y discusión del Plan de Acompañamiento de $A+S$, UC, en el marco del proyecto Uniservitate (figura 1 ), se resuelve con la elaboración de un modelo de acompañamiento e institucionalización de aprendizajeservicio (previa aplicación de instrumento) que permite abordar las principales aristas que involucra la metodología, sus principios y fundamentos, aplicación de los conocimientos adquiridos.

Figura 1. Plan de acompañamiento de institucionalización de $A+S$, Latinoamérica y el Caribe

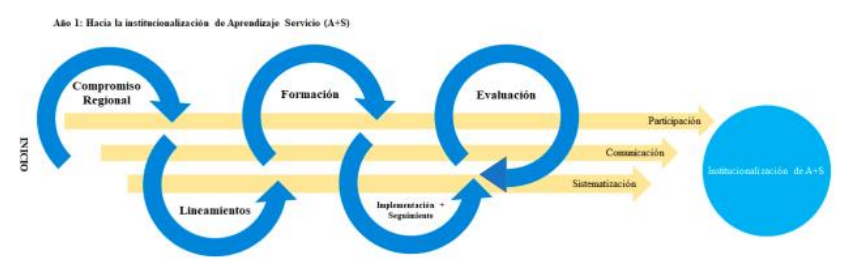

Fuente: elaboración propia
Se involucra a docentes y unidades de apoyo de cada universidad participante, además de comprender que nuestro propósito es impactar desde la educación construida con el otro y la otra en Latinoamérica con Latinoamérica. Es importante mencionar, que las directrices que aporta este modelo se basan en parámetros generales, ya que debemos reconocer la identidad, lineamientos y propósitos que cada casa de estudio posee y desde ahí construir las mejores opciones y vías de implementación del aprendizaje-servicio, que sea coherente con la visión y misión de las universidades y responda a la labor educativa y social que defendemos desde $A+S$.

Previo a la elaboración del Plan, se desarrolló un diagnóstico que identifica diversos desafíos según las dimensiones de la rúbrica. En este artículo, mostramos los resultados de las subdimensiones que se encuentran en una etapa "Inicial" en las que el Plan de Acompañamiento ha podido generar acciones rápidas debido a la experiencia de la UC y en donde puede mostrar avance o futuras acciones concretas para ser desarrolladas en las 3 universidades acompañadas. En la dimensión 2 "Docentes $A+S$ ", se identificó que la subdimensión "capacitación de la metodología" sería una acción a implementar debido a la experiencia que posee el programa $A+S$, UC, en la implementación de espacios formativos de aprendizajeservicio (curso de formación continua y los talleres para docentes). En la dimensión 3, no se cuenta con alguna subdimensión en que todas declaren 
estar en el Inicio. Más bien, podemos destacar el desarrollo de UCA, PUCE y PUJ en la subdimensión "Vinculación recíproca y duradera entre socios y la universidad" por la importancia que tiene la comunidad en los proyectos de aprendizaje-servicio. En la dimensión 4, "Protagonismo estudiantil" se refleja que se requiere fortalecer la "sensibilización e información que posee el estudiantado sobre aprendizaje-servicio" y el "Liderazgo del estudiantado y participación en instancias donde se aborda la metodología aprendizaje-servicio", ámbitos en que se requieren definir prontamente acciones concretas. En la dimensión 5 "Soporte y gestión de la implementación de $A+S$ en la Unidad Académica", se observó que la subdimensión "Encargado $A+S$ en la unidad académica" es un aspecto considerado relevante para los equipos acompañados. Dentro de la misma dimensión, en las subdimensiones "Mecanismos de evaluación y seguimiento" y "Difusión de experiencias de aprendizaje-servicio" existirán mecanismos para fortalecer estos ámbitos, por los instrumentos que se elaboren en el marco del proyecto y por los espacios académicos que se implementen durante el proyecto (ejemplo el simposio anual denominado Uniservitate).

Un aspecto relevante en la elaboración de un modelo es que se aplique y ello va acompañado de acciones, compromisos y responsabilidades de quienes participan del proceso. Es entonces que iniciamos su implementación con dos hitos fundamentales para el desarrollo y ejecución del modelo. El primero de ellos, fue realizar la invitación a las universidades participantes del proceso a asumir un "Compromiso Regional" que como HUB LATAM podamos contribuir desde los proyectos de aprendizaje-servicio que se están desarrollando y los que se implementarán en el futuro contribuyendo - en parte a la reducción de brechas y accesos que son producto de las desigualdades que tenemos en la región, a la promoción y posicionamiento de la riqueza desde la interculturalidad que posee Latinoamérica, y responder al llamado que nos hace Fratelli Tutti (Papa Francisco, 2020) a construir nuestras relaciones humanas desde una mirada sustentable, colaborativa y transformadora para sociedades que promueven la dignidad y justicia.

Posteriormente, se revisó la literatura sobre la experiencia que la UC ha desarrollado en sus 17 años de experiencias con aprendizaje-servicio, particularmente, en la universidad, destacando, entre otros, el apoyo de las autoridades desde Rectoría a jefaturas de las distintas unidades académicas, el acompañamiento pedagógico con el foco en la calidad docente, la elaboración de un modelo de implementación de aprendizaje-servicio en el aula que permita flexibilidad, pero a la vez profundidad en la implementación de aprendizaje-servicio y una propuesta de modelo de institucionalización de aprendizajeservicio en las carreras (Montalva, et al., 2019). Esta experiencia se ha transmitido en las instancias que hemos compartido con los equipos y la red 
global Uniservitate.

El plan de acompañamiento se compone de 4 etapas. La primera de ellas es la etapa de "Lineamientos", la cual tiene por objetivo hacer una nivelación de expectativas y presentar cuáles serán los objetivos y actividades por desarrollar durante el proceso de acompañamiento a las universidades. Es por ello que la participación de autoridades como rectorías las consideramos una parte fundamental en este proceso, ya que se deben transformar en promotoras de aprendizaje-servicio como una de las vías para la formación profesional integral, inclusiva y transformadora. Es entonces cómo logramos una reunión entre 4 de los 5 rectores involucrados en el proyecto con el objetivo de socializar los lineamientos centrales de la metodología aprendizaje-servicio, esperando contar con el apoyo de las autoridades de las IESC. Luego, también, se han realizado presentaciones a equipos académicos clave de las universidades acompañadas, compartiendo la experiencia de la UC en un espacio para un diálogo más directo sobre la metodología. Una segunda etapa del modelo es la de "Formación": este ítem comprende el diseño de un curso online, instancia formativa que tiene por objetivo profundizar en los principales hitos, principios y fundamentos de la metodología en el contexto de su implementación: su relación con la misión de la IES, la vinculación con la comunidad, la factibilidad, espiritualidad, reflexión y la evaluación de aprendizajes. En su primera versión (junio - agosto) 24 participantes, entre docentes y profesionales de apoyo de unidades de vinculación con el medio o compromiso social que se verán involucrados en sostener los proyectos y unidades de aprendizaje-servicio en las universidades acompañadas. Es importante mencionar que ya está en curso una segunda versión (septiembre - noviembre) llegando a cerca de 50 participantes durante este 2021 que tendrán una formación en aprendizajeservicio. Una tercera etapa es la "Implementación y Seguimiento", esto dice relación con el diseño de acciones para realizar un acompañamiento a docentes que van a implementar la metodología que refuercen algunos lineamientos sobre hitos como la reflexión, la relación con la comunidad, la espiritualidad. De forma complementaria, se comparten en la plataforma colaborativa Slack herramientas y recursos que puedan fortalecer la calidad del proceso, como por ejemplo el "Kit de Herramientas para tus cursos aprendizaje-servicio", "la Guía de Institucionalización A+S UC", y la invitación permanente a espacios como webminars y talleres. Finalmente, la etapa de "Evaluación", implica realizar un cierre de reconocimiento para quienes participaron en los proyectos, además de establecer mecanismos $\mathrm{y} / \mathrm{o}$ instrumentos de evaluación de percepción y aprendizajes tanto a nivel cualitativo como cuantitativo que tienen como propósito fortalecer los procesos de implementación e institucionalización de aprendizajeservicio al interior de las universidades.

El plan de acompañamiento, se co- 
construyó con un espíritu de colaboración y trabajo en equipo con las universidades acompañadas. De allí, que se debe reconocer que cada universidad tiene un contexto propio que ha sido considerado para la coconstrucción de un acompañamiento de etapas que han sido diseñadas de forma genérica.

En relación a la literatura, este plan considera una definición de institucionalización que busca integrar la metodología en la estructura de las universidades acompañadas (Jouannet et al., 2015) e incorpora una propuesta de definición institucional que puede guiar la implementación de $A+S$ en los próximos años (Furco y Billig, 2002). Con esto se busca fortalecer las principales dimensiones que suponen una institucionalización de la metodología, a partir de la rúbrica de Furco (2011) y la adaptación realizada por la UC (Caire et al., 2018). La experiencia de la universidad ha sido importante para crear el plan, a partir de su modelo de institucionalización en las carreras (Jouannet et al., 2015) que incluye un proceso con actividades que se han realizado: la capacitación a docentes, la difusión de experiencias, la participación de las autoridades, entre otros.

En específico, el plan también recoge aspectos relevantes del modelo de implementación de $\mathrm{A}+\mathrm{S}$, UC, (Jouannet, et al., 2013), como el hito de la reflexión (Montalva, et al., 2015) y la sociedad que debe existir entre la universidad y la comunidad. Además, pone en discusión la importancia de un hito que aborde la dimensión espiritual representada por la identidad de las universidades y el encuentro que se produce entre las comunidades, los equipos docentes y estudiantes (Pete, 2020).

\section{Conclusiones}

Como se puede observar en la discusión, hay elementos que se consolidan de manera robusta tras la elaboración y ejecución del Plan de Acompañamiento, estos coinciden con los ejes principales del plan de institucionalización del aprendizajeservicio en la UC como orientador de las acciones a desarrollar, pero que sin embargo, van adquiriendo una personalidad propia al ser implementados en cada universidad acompañada. La mirada desde las y los docentes que intervienen en cada una de las instancias que se promueven desde el HUB LATAM a cargo de UC, así también como las que convoca Uniservitate.

La importancia del trabajo colaborativo en el diseño conjunto con las universidades participantes generan una ejecución del modelo de rápidos avances, - que por lo demás lo compone una gran calidez humana a pesar de la virtualidad, se han creado lazos y posibles proyectos en conjuntoen donde la barrera del idioma (UNICAP, Brasil) no ha sido un obstáculo sino una instancia de aprendizaje e interés por las diferentes culturas del espacio que construimos a raíz del curso de formación. Cabe destacar dos aspectos relevantes y tal como se mencionó anteriormente, el proceso de formación docente, los recursos para docentes, estudiantes, socios comunitarios y cualquier persona

Del Valle, F., Fontana, R. y Sepúlveda, J. (2021). Un compromiso para la transformación social: el plan UC para consolidar a A+S en América Latina. RIDAS, Revista Iberoamericana de Aprendizaje Servicio, 12, 80-90. DOI10.1344/RIDAS2021.12.9 
interesada en transformar e innovar en educación.

Sin duda, tenemos el desafío de que colaborativamente podamos construir capacidades para que el aprendizajeservicio sea sostenible en las instituciones de educación superior católicas en el tiempo, sus acciones y convicciones. El desafío es enorme siendo que existen áreas como el protagonismo estudiantil, las habilidades transversales y la gestión de los proyectos debe contemplar acciones presentes y futuras. Sin embargo, concluimos que hemos avanzado con una riqueza cultural y generosidad que permitirá avanzar en estos desafíos.

La idea de potenciar el proyecto Uniservitate y posicionar la metodología de enseñanza aprendizaje posibilitando el encuentro de saberes; el protagonismo estudiantil con y desde el territorio; la contribución a la coconstrucción de soluciones a problemáticas y la innovación en la forma en que compartimos el conocimiento es la que hacen del aprendizaje-servicio, una alternativa para la democratización del conocimiento y, como consecuencia, sociedades que promueven valores como la justicia, la equidad y la tolerancia.

\section{Agradecimientos}

A los equipos de las 4 universidades acompañadas de quienes hemos ido aprendiendo en todos los espacios que hemos compartido.

Al equipo del Centro de Desarrollo
Docente UC, especialmente, a quienes han apoyado el trabajo del equipo del Nodo Regional Latinoamérica.

\section{Referencias bibliográficas}

Caire, M., González, M., Jouannet, C., Montalva, J., y Ponce, C. (2018). Guía para la institucionalización de Aprendizaje Servicio en una carrera. Centro de Desarrollo Docente. Pontificia Universidad Católica de Chile. https://cld.bz/skUYaPu

Furco, A., \& Billig, S. H. (2002). Service-Learning: The Essence of Pedagogy. Information Age Publishing.

Furco, A. (2011). Rúbrica de autoevaluación para la institucionalización del aprendizajeservicio en la educación superior (revisión 2003). Educación Global Research, (0), 77-88.

http://educaciocp89.webjoomla.es/wpcontent/uploads/04-Furco-2Castellano.pdf

Caire, M., González, M., y Jouannet, C. (2019). Rúbrica para la institucionalización de aprendizaje servicio en una carrera. Actas de la $\checkmark$ Jornada de investigadores sobre Aprendizaje-Servicio (pp. 57-62). CLAYSS-Red Iberoamericana de aprendizaje-servicio. https://www.clayss.org.ar/JIAS/V ji as/Libro VJIA-S.pdf

Jouannet, C., Salas, M., y Contreras, M. (2013). Modelo de implementación de aprendizaje servicio $(A+S)$ en la UC.

Del Valle, F., Fontana, R. y Sepúlveda, J. (2021). Un compromiso para la transformación social: el plan UC para consolidar a A+S en América Latina. RIDAS, Revista Iberoamericana de Aprendizaje Servicio, 12, 80-90. DOI10.1344/RIDAS2021.12.9 
Una experiencia que impacta positivamente en la formación profesional integral. Calidad en la Educación, (39), 198-212.

http://dx.doi.org/10.4067/S071845652013000200007

Jouannet, C., Montalva. J.T., Ponce, C., y Von Borries, V. (2015). Diseño de un modelo de institucionalización de la metodología de aprendizaje servicio en educación superior. RIDAS, Revista Iberoamericana de Aprendizaje y Servicio, (1) , 112131.

http://dx.doi.org/10.1344/ridas 2015 .1 .7

Montalva, J., Jouannet, C., González, S., y O'Ryan, L. (2019). Aprendizaje Servicio en la Pontificia Universidad Católica de Chile ( $A+S$ UC). Un camino para el desarrollo de ciudadanos comprometidos. En B. Hasbún y V. Pizarro. (Eds.), Aprendizaje Servicio en la educación superior chilena (pp. 48-55). Ediciones FEA-FEN Universidad de Chile.

https://libros.uchile.cl/files/presses/ $1 /$ monographs/953/submission/proo $\mathrm{f} / 48 /$ index.html\#zoom $=\mathrm{z}$

Montalva, J., Ponce, C., Jouannet, C., y Jara, M. (2015). Elementos clave para una reflexión de calidad. Actas de la III Jornada de investigadores sobre AprendizajeServicio (pp. 59-62). CLAYSS-Red Iberoamericana de aprendizajeservicio.

Papa Francisco. (3 de octubre de 2020). Carta Encíclica Fratelli Tutti del Santo Padre Francisco sobre la
Fraternidad y la Amistad Social.

https://www.vatican.va/content/fran cesco/es/encyclicals/documents/pap a-francesco 20201003 enciclicafratelli-tutti.html

Pete, J. (2020). Spirituality for Service Learning. Tangaza University College.

Del Valle, F., Fontana, R. y Sepúlveda, J. (2021). Un compromiso para la transformación social: el plan UC para consolidar a A+S en América Latina. RIDAS, Revista Iberoamericana de Aprendizaje Servicio, 12, 80-90. DOI10.1344/RIDAS2021.12.9 